\title{
General Caching Is Hard: Even with Small Pages ${ }^{\star}$
}

\author{
Lukáš Folwarczný and Jiří Sgall \\ Computer Science Institute of Charles University, \\ Faculty of Mathematics and Physics, \\ Malostranské nám. 25, CZ-11800 Praha 1, \\ Prague, Czech Republic. \\ \{folwar, sgall\}@iuuk.mff.cuni.cz
}

\begin{abstract}
Caching (also known as paging) is a classical problem concerning page replacement policies in two-level memory systems. General caching is the variant with pages of different sizes and fault costs. We give the first NP-hardness result for general caching with small pages: General caching is (strongly) NP-hard even when page sizes are limited to $\{1,2,3\}$. It holds already in the fault model (each page has unit fault cost) as well as in the bit model (each page has the same fault cost as size). We also give a very short proof of the strong NP-hardness of general caching with page sizes restricted to $\{1,2,3\}$ and arbitrary costs.
\end{abstract}

Keywords: general caching, small pages, NP-hardness

\section{Introduction}

Caching (also known as uniform caching or paging) is a classical problem in the area of online algorithms and has been extensively studied since 1960s. It models a two-level memory system: There is the fast memory of size $C$ (the cache) and a slow but large main memory where all data reside. The problem instance comprises a sequence of requests, each demanding a page from the main memory. No cost is incurred if the requested page is present in the cache (a cache hit). If the requested page is not present in the cache (a cache fault), the page must be loaded at the fault cost of one; some page must be evicted to make space for the new one when there are already $C$ pages in the cache. The natural objective is to evict pages in such a way that the total fault cost is minimized. For a reference on classical results, see Borodin and El-Yaniv [6].

In 1990s, with the advent of World Wide Web, a generalized variant called file caching or simply general caching was studied $[9,10]$. In this setting, each page $p$ has its $\operatorname{SizE}(p)$ and $\operatorname{CosT}(p)$. It costs $\operatorname{COsT}(p)$ to load this page into the cache and the page occupies $\operatorname{SIZE}(p)$ units of memory there. Uniform caching is the special case satisfying $\operatorname{SIzE}(p)=\operatorname{COST}(p)=1$ for every page $p$. Other important cases of this general model are

\footnotetext{
* Partially supported by the Center of Excellence - Institute for Theoretical Computer Science, Prague (project P202/12/G061 of GA ČR).
} 
- the cost model (weighted caching): $\operatorname{SIZE}(p)=1$ for every page $p$;

- the bit model: $\operatorname{COST}(p)=\operatorname{SIZE}(p)$ for every page $p$;

- the fault model: $\operatorname{CosT}(p)=1$ for every page $p$.

General caching is still a thriving area of research as may be documented by the fact that a randomized online algorithm with the optimal asymptotic competitive ratio $\mathcal{O}(\log C)$ was given only recently by Adamaszek et al. [1]; this article may also serve as a reference on the current state of knowledge.

Caching, as described so far, requires the service to load the requested page when a fault occurs, which is known as caching under the forced policy. Allowing the service to pay the fault cost without actually loading the requested page to the cache gives another useful and studied variant of caching, the optional policy.

Previous work. In this article, we consider the problem of finding the optimal service in the offline version of caching when the whole request sequence is known in advance. Uniform caching is solvable in polynomial time with a natural algorithm known as Belady's rule [5]. Caching in the cost model is a special case of the $k$-server problem and is also solvable in polynomial time [7]. In late 1990s, the questions about the complexity status of general caching were raised. The situation was summed up by Albers et al. [2]: "The hardness results for caching problems are very inconclusive. The NP-hardness result for the Bit model uses a reduction from PARTITION, which has pseudopolynomial algorithms. Thus a similar algorithm may well exist for the Bit model. We do not know whether computing the optimum in the Fault model is NP-hard."

There was no improvement until a breakthrough in 2010 when Chrobak et al. [8] showed that general caching is strongly NP-hard, already in the case of the fault model as well as in the case of the bit model. General caching is usually studied under the assumption that the largest page size is very small in comparison with the total cache size, as is for example the case of the aforementioned article by Albers et al. [2]. Instances of caching with pages larger than half of the cache size (so called obstacles) are required in the proof given by Chrobak et al. Therefore, this hardness result is in fact still quite inconclusive.

Contribution. We give a novel proof of strong NP-hardness for general caching which gives the first hardness result restricted to small pages:

Theorem 1.1. General caching is strongly NP-hard even in the case when the page sizes are limited to $\{1,2,3\}$, for both the fault model and the bit model, and under each of the forced and optional policies.

The proof of the result for general costs (and sizes $\{1,2,3\}$ ) is rather simple, in particular significantly simpler than the one given by Chrobak et al. [8]. The reductions for the result in the fault and bit models are significantly more involved and require a non-trivial potential-function-like argument.

Relation to interval packing. As observed by Chrobak et al. [8], there is a tight connection between caching under the optional policy and the problem called interval packing. A set of weighted intervals together with a limit $W$ is given in the problem and the target is either to (a) choose a subset of intervals of the maximum cardinality such that at each point the total weight of intersected 
intervals is limited by $W$ (corresponds to caching in the fault model), or (b) choose a subset of intervals of the maximum total weight such that at each point the total weight of intersected intervals is limited by $W$ (corresponds to the bit model). The problem is a special case of the unsplittable flow problem on line graphs [3]. Our proofs can be considered as proofs of strong NP-hardness of either version of interval packing. See Chrobak et al. [8] for details on the connection between interval packing and caching.

Open problems. We prove that general caching with page sizes $\{1,2,3\}$ is strongly NP-hard while general caching with unit page sizes is easily polynomially solvable. Closing the gap will definitely contribute to a better understanding of the problem:

Question 1.2. Is general caching also (strongly) NP-hard when page sizes are limited to $\{1,2\}$ ? Can caching with page sizes $\{1,2\}$ be solved in polynomial time, at least in the bit or fault model?

In a broader perspective, the complexity status of general caching is still far from being well understood as the best known result on approximation is a 4approximation due to Bar-Noy et al. [4] and there is no result on the hardness of approximation. Therefore, better understanding of the complexity of general caching remains an important challenge.

Question 1.3. Is there an algorithm for general caching with an approximation ratio better than 4, or even a PTAS? Is general caching APX-hard?

Outline. The main part of our work - a polynomial-time reduction from independent set to caching in the fault model under the optional policy with page sizes restricted to $\{1,2,3\}$ - is explained in Section 2 and its validity is proven in Section 3. In Section 4, we show how to modify the reduction so that it works for the bit model as well. In Section 5, we show how to obtain the hardness results also for the forced policy. Finally, we give a self-contained presentation of the simple proof of strong NP-hardness for general costs (in fact, only two different and polynomial costs are needed) in Appendix A.

\section{Reduction}

The decision problem InDEPENDENTSET is well-known to be NP-complete. By 3CACHING(FORCED) and 3CACHING(OPTIONAL) we denote the decision versions of caching under each policy with page sizes restricted to $\{1,2,3\}$.

Problem: InDEPEndentSet

Instance: A graph $G$ and a number $K$.

Question: Is there an independent set of cardinality $K$ in $G$ ? 


\section{Problem: 3CACHING(policy)}

Instance: A universe of pages, a sequence of page requests, numbers $C$ and $L$. For each page $p$ it holds $\operatorname{SizE}(p) \in\{1,2,3\}$.

Question: Is there a service under the policy policy of the request sequence using the cache of size $C$ with a total fault cost of at most $L$ ?

We define 3CACHIng(FAUlt,policy) to be the problem 3CACHING(policy) with the additional requirement that page costs adhere to the fault model. The problem 3CACHING(BIT,policy) is defined analogously.

In this section, we describe a polynomial-time reduction from INDEPENDENTSET to 3CACHING(FAUlt, OPTIONAL). Informally, a set of pages of size two and three is associated with each edge and a page of size one is associated with each vertex. Each vertex-page is requested only twice while there are many requests on pages associated with edges. The request sequence is designed in such a way that the number of vertex-pages that are cached between the two requests in the optimal service is equal to the the size of the maximum independent set.

We now show the request sequence of caching corresponding to the graph given in InDEPENDENTSET with a parameter $H$. In the next section, we prove that it is possible to set a proper value of $H$ and a proper fault cost limit $L$ such that the reduction becomes a valid polynomial-time reduction.

Reduction 2.1. Let $G=(V, E)$ be the instance of IndependentSet. The graph $G$ has $n$ vertices and $m$ edges and there is an arbitrary fixed order of edges $e_{1}, \ldots, e_{m}$. Let $H$ be a parameter bounded by a polynomial function of $n$.

A corresponding instance $\mathcal{I}_{G}$ of $3 \mathrm{CACHING}$ (FAULT, OPTIONAL) is an instance with the cache size $C=2 m H+1$ and the total of $6 m H+n$ pages. The structure of the pages and the requests sequence is described below.

Pages. For each vertex $v$, we have a vertex-page $p_{v}$ of size one. For each edge $e$, there are $6 H$ edge-pages associated with it that are divided into $H$ groups. The $i$ th group consists of six pages $\bar{a}_{i}^{e}, \alpha_{i}^{e}, a_{i}^{e}, b_{i}^{e}, \beta_{i}^{e}, \bar{b}_{i}^{e}$ where pages $\alpha_{i}^{e}$ and $\beta_{i}^{e}$ have size three and the remaining four pages have size two.

For a fixed edge $e$, let $\bar{a}^{e}$-pages be all pages $\bar{a}_{i}^{e}$ for $i=1, \ldots, H$. Let also $\bar{a}$-pages be all $\bar{a}^{e}$-pages for $e=e_{1}, \ldots, e_{m}$. The remaining collections of pages $\left(\alpha^{e}\right.$-pages, $\alpha$-pages, $\left.\ldots\right)$ are defined in a similar fashion.

Request sequence. The request sequence of $\mathcal{I}_{G}$ is organized in phases and blocks. There is one phase for each vertex $v \in V$, we call such a phase the $v$ phase. There are exactly two requests on each vertex-page $p_{v}$, one just before the beginning of the $v$-phase and one just after the end of the $v$-phase; these requests do not belong to any phase. The order of phases is arbitrary. In each $v$ phase, there are $2 H$ adjacent blocks associated with every edge $e$ incident with $v$; the blocks for different incident edges are ordered arbitrarily. In addition, there is one initial block $I$ before all phases and one final block $F$ after all phases. Altogether, there are $d=4 m H+2$ blocks.

Let $e=\{u, v\}$ be an edge, let us assume that the $u$-phase precedes the $v$-phase. The blocks associated with $e$ in the $u$-phase are denoted by $B_{1,1}^{e}$, 
$B_{1,2}^{e}, \ldots, B_{i, 1}^{e}, B_{i, 2}^{e}, \ldots, B_{H, 1}^{e}, B_{H, 2}^{e}$, in this order, and the blocks in the $v$-phase are denoted by $B_{1,3}^{e}, B_{1,4}^{e}, \ldots, B_{i, 3}^{e}, B_{i, 4}^{e}, \ldots, B_{H, 3}^{e}, B_{H, 4}^{e}$, in this order. An example is given in Fig. 1.

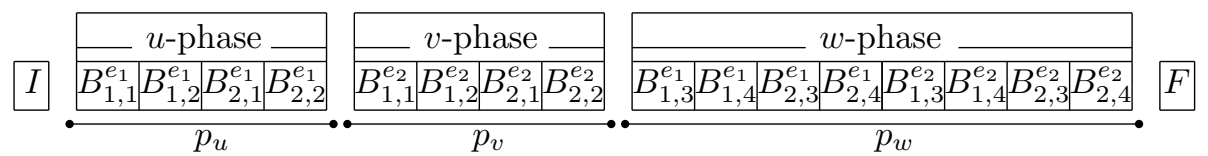

Fig. 1. An example of phases, blocks and requests on vertex-pages for a graph with three vertices $u, v, w$ and two edges $e_{1}=\{u, w\}, e_{2}=\{v, w\}$ when $H=2$

Even though each block is associated with some fixed edge, it contains one or more requests to the associated pages for every edge $e$. In each block, we process the edges in the order $e_{1}, \ldots, e_{m}$ that was fixed above. Pages associated with the edge $e$ are requested in two rounds. In each round, we process groups $1, \ldots, H$ in this order. When processing the $i$ th group of the edge $e$, we request one or more pages of this group, depending on the block we are in. Table 1 determines which pages are requested.

Table 1. Requests associated with an edge $e$

\begin{tabular}{|c|ccc|}
\hline Block & First round & • Second round \\
\hline before $B_{i, 1}^{e}$ & $\bar{a}_{i}^{e}$ & $\bullet$ & \\
\hline$B_{i, 1}^{e}$ & $\bar{a}_{i}^{e}, \alpha_{i}^{e}$ & $\bullet$ & $b_{i}^{e}$ \\
\hline$B_{i, 2}^{e}$ & $\alpha_{i}^{e}, a_{i}^{e}$ & $\bullet$ & $b_{i}^{e}$ \\
\hline between $B_{i, 2}^{e}$ and $B_{i, 3}^{e}$ & $a_{i}^{e}$ & $\bullet$ & $b_{i}^{e}$ \\
\hline$B_{i, 3}^{e}$ & $a_{i}^{e}$ & $\bullet$ & $b_{i}^{e}, \beta_{i}^{e}$ \\
\hline$B_{i, 4}^{e}$ & $a_{i}^{e}$ & $\bullet$ & $\beta_{i}^{e}, \bar{b}_{i}^{e}$ \\
\hline after $B_{i, 4}^{e}$ & & $\bullet$ & $\bar{b}_{i}^{e}$ \\
\hline
\end{tabular}

Reduction 2.1 is now complete. An example of requests on edge-pages associated with one edge $e$ is depicted in Fig. 2. Notice that the order of the pages associated with $e$ is the same in all blocks; more precisely, in each block the requests on the pages associated with $e$ form a subsequence of

$$
\bar{a}_{1}^{e} \alpha_{1}^{e} a_{1}^{e} \ldots \bar{a}_{i}^{e} \alpha_{i}^{e} a_{i}^{e} \ldots \bar{a}_{H}^{e} \alpha_{H}^{e} a_{H}^{e} b_{1}^{e} \beta_{1}^{e} \bar{b}_{1}^{e} \ldots b_{i}^{e} \beta_{i}^{e} \bar{b}_{i}^{e} \ldots b_{H}^{e} \beta_{H}^{e} \bar{b}_{H}^{e} .
$$

Preliminaries for the proof. Instead of minimizing the service cost, we maximize the savings compared to the service which does not use the cache at all. This is clearly equivalent when considering the decision versions of the problems.

Without loss of generality, we may assume that any page is brought into the cache only immediately before some request to that page and removed from the cache only after some (possibly different) request to that page; furthermore, at the beginning and at the end the cache is empty. I.e., a page may be in the cache 
only between two consecutive requests to this page, and either remains in the cache for the whole interval or not at all.

Each page of size three is requested only twice in two consecutive blocks, and these blocks are distinct for all pages of size three. Thus, a service of edge-pages is valid if and only if at each time, at most $m H$ edge-pages are in the cache. It is convenient to think of the cache as of $m H$ slots for edge-pages.

As each vertex-page is requested twice, the savings on the $n$ vertex-pages are at most $n$. Furthermore, a vertex-page can be cached if and only if during the phase it never happens that at the same time all slots for edge-pages are full and a page of size three is cached.

Let $S_{B}$ denote the set of all edge-pages cached at the beginning of the block $B$ and let $S_{B}^{e}$ be the set of pages in $S_{B}$ associated with the edge $e$. We use $s_{B}=\left|S_{B}\right|$ and $s_{B}^{e}=\left|S_{B}^{e}\right|$ for the sizes of the sets. Each edge-page is requested only in a contiguous segment of blocks, once in each block. It follows that the total savings on edge-pages are equal to $\sum_{B} s_{B}$ where the sum is over all blocks. In particular, the maximal possible savings on the edge-pages are $(d-1) \mathrm{mH}$, using the fact that $S_{I}$ is empty. We shall show that the maximum savings are $(d-1) m H+K$ where $K$ is the size of the maximum independent set in $G$.

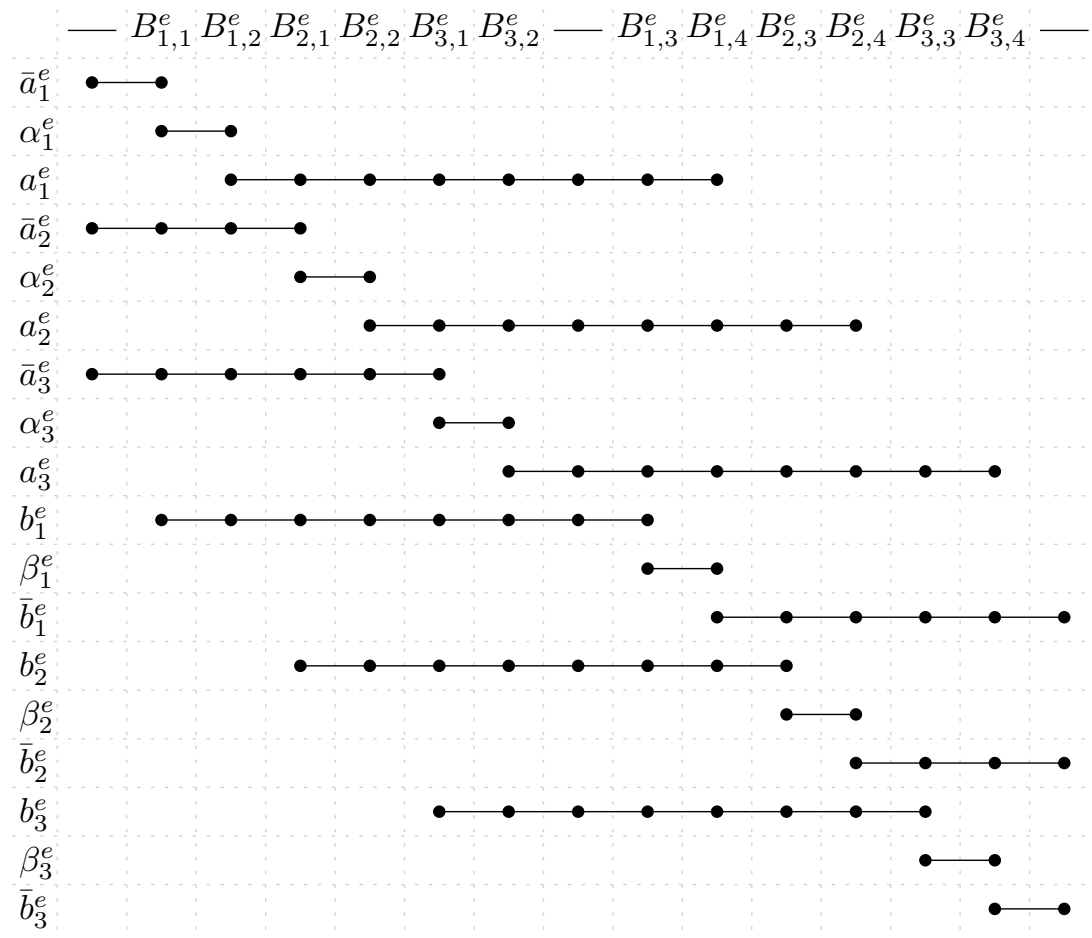

Fig. 2. Requests on all pages associated with the edge $e$ when $H=3$. Each column represents some block(s). The labelled columns represent the blocks in the heading, the first column represents every block before $B_{1,1}^{e}$, the middle column represents every block between $B_{3,2}^{e}$ and $B_{1,3}^{e}$, and the last column represents every block after $B_{3,4}^{e}$. The requests in one column are ordered from top to bottom. 
Almost-fault model. To understand the reduction, we consider what happens if we relax the requirements of the fault model and set the cost of each vertexpage to $1 /(n+1)$ instead of 1 as required by the fault model.

In this scenario, the total savings on vertex-pages are $n /(n+1)<1$ which is less than savings incurred by one edge-page. Therefore, edge-pages must be served optimally in the optimal service of the whole request sequence.

In this case, the reduction works already for $H=1$. This leads to a quite short proof of the strong NP-hardness for general caching and we give this proof in Appendix A. Here, we show just the main ideas that are important also for the design of our caching instance in the fault and bit models.

We first prove that for each edge $e$ and each block $B \neq I$ we have $s_{B}^{e}=1$ (see Appendix A). Using this we show below that for each edge $e$, at least one of the pages $\alpha_{1}^{e}$ and $\beta_{1}^{e}$ is cached between its two requests. This implies that the set of all vertices $v$ such that $p_{v}$ is cached between its two requests is independent.

For a contradiction, let us assume that for some edge $e$, neither of the pages $\alpha_{1}^{e}$ and $\beta_{1}^{e}$ is cached between its two requests. Because pages $\alpha_{1}^{e}$ and $\beta_{1}^{e}$ are forbidden, there is $b_{1}^{e}$ in $S_{B_{1,2}^{e}}$ and $a_{1}^{e}$ in $S_{B_{1,3}^{e}}$. Somewhere between these two blocks $B_{1,2}^{e}$ and $B_{1,3}^{e}$, we must switch from caching $b_{1}^{e}$ to caching $a_{1}^{e}$. However, this is impossible, because the order of requests implies that we would have to cache both $b_{1}^{e}$ and $a_{1}^{e}$ at some moment (see Fig. 3). However, there is no place in the cache for such an operation, as $s_{B}^{e}=1$ for every $e^{\prime}$ and $B \neq I$.

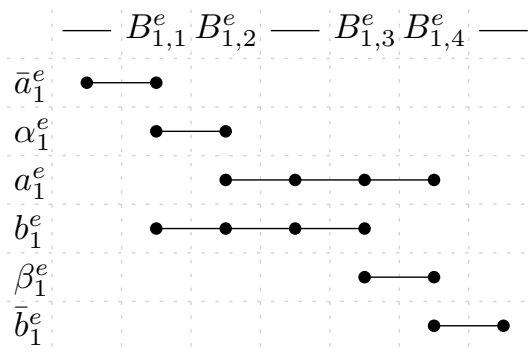

Fig. 3. Pages associated with one edge when $H=1$

In the fault model, the corresponding claim $s_{B}^{e}=H$ does not hold. Instead, we prove that the value of $s_{B}^{e}$ cannot change much during the service and when we use $H$ large enough, we still get a working reduction.

\section{Proof of Correctness}

In this section, we show that the reduction described in the previous section is indeed a reduction from INDEPENDENTSET set to 3CACHING(FAULT,OPTIONAL). We prove that there is an independent set of cardinality $K$ in $G$ if and only if there is a service of the caching instance $\mathcal{I}_{G}$ with the total savings of at least $(d-1) m H+K$. First the easy direction, which holds for any value of the parameter $H$. 
Lemma 3.1. Let $G$ be a graph and $\mathcal{I}_{G}$ the corresponding caching instance from Reduction 2.1. Suppose that there is an independent set $W$ of cardinality $K$ in $G$. Then there exists a service of $\mathcal{I}_{G}$ with the total savings of at least $(d-1) m H+K$.

Proof. For any edge $e$, denote $e=\{u, v\}$ so that the $u$-phase precedes the $v$ phase. If $u \in W$, we keep all $\bar{a}^{e}$-pages, $b^{e}$-pages, $\beta^{e}$-pages and $\bar{b}^{e}$-pages in the cache from the first to the last request on each page, but we do not cache $a^{e}$-pages and $\alpha^{e}$-pages at any time. Otherwise, we cache all $\bar{a}^{e}$-pages, $\alpha^{e}$-pages, $a^{e}$-pages and $\bar{b}^{e}$-pages, but do not cache $b^{e}$-pages and $\beta^{e}$-pages at any time. Fig. 4 shows these two cases for the first group of pages. In both cases, at each time at most one page associated with each group of each edge is in the cache and the savings on those pages are $(d-1) m H$. We know that the pages fit in the cache because of the observations made in Section 2.

For any $v \in W$, we cache $p_{v}$ between its two requests. To check that this is a valid service, observe that if $v \in W$, then during the corresponding phase no page of size three is cached. Thus, the page $p_{v}$ always fits in the cache together with at most $m H$ pages of size two.

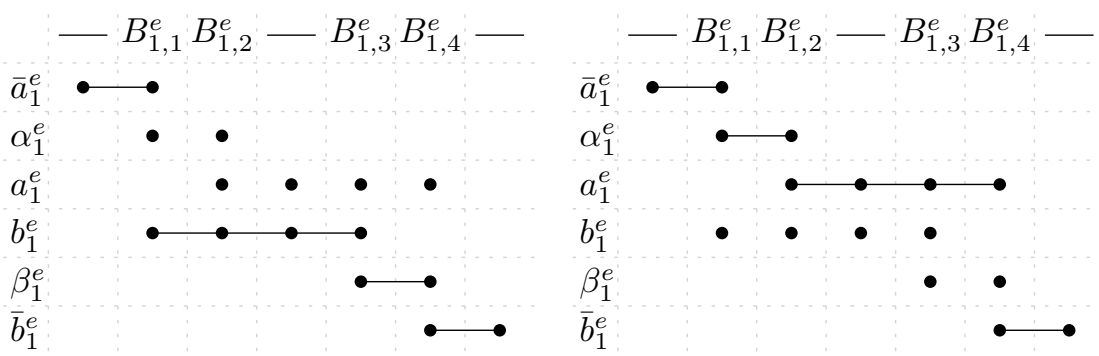

Fig. 4. The two ways of caching in Lemma 3.1

We prove the converse in a sequence of lemmata. In Section 4, we will show how to reuse the proof for the bit model. To be able to do that, we list explicitly all the assumptions about the caching instance that are used in the following proofs.

Properties 3.2. Let $\mathcal{T}_{G}$ be an instance of general caching corresponding to a graph $G=(V, E)$ with $n$ vertices, $m$ edges $e_{1}, \ldots, e_{m}$, the same cache size and the same universe of pages as in Reduction 2.1. The request sequence is again split into phases, one phase for each vertex. Each phase is again partitioned into blocks, there is one initial block $I$ before all phases and one final block $F$ after all phases. There is the total of $d$ blocks.

The instance $\mathcal{T}_{G}$ is required to fulfill the following list of properties:

(a) Each vertex page $p_{v}$ is requested exactly twice, right before the $v$-phase and right after the $v$-phase.

(b) The total savings incurred on edge-pages are equal to $\sum s_{B}$ (summing over all blocks).

(c) For each edge $e$, there are exactly $H$ pages associated with $e$ requested in $I$, all the $\bar{a}^{e}$-pages, and exactly $H$ pages associated with $e$ requested in $F$, all the $\bar{b}^{e}$-pages. 
Table 2. Request sequences on all pages associated with an edge $e$

\begin{tabular}{|c|c|}
\hline Block & First round • Second round \\
\hline before $B_{1,1}^{e}$ & $\bar{a}_{1}^{e} \ldots \bar{a}_{H}^{e} \bullet$ \\
$B_{i, 1}^{e}$ & $a_{1}^{e} \ldots a_{i-1}^{e} \bar{a}_{i}^{e} \alpha_{i}^{e} \bar{a}_{i+1}^{e} \bar{a}_{i+2}^{e} \ldots \bar{a}_{H}^{e} \cdot b_{1}^{e} \ldots b_{i}^{e}$ \\
$B_{i, 2}^{e}$ & $a_{1}^{e} \ldots a_{i-1}^{e} \alpha_{i}^{e} a_{i}^{e} \bar{a}_{i+1}^{e} \bar{a}_{i+2}^{e} \ldots \bar{a}_{H}^{e} \cdot b_{1}^{e} \ldots b_{i}^{e}$ \\
between $B_{H, 2}^{e}$ and $B_{1,3}^{e}$ & $a_{1}^{e} \ldots a_{H}^{e} \cdot b_{1}^{e} \ldots b_{H}^{e}$ \\
$B_{i, 3}^{e}$ & $a_{i}^{e} \ldots a_{H}^{e} \cdot \bar{b}_{1}^{e} \ldots \bar{b}_{i-1}^{e} b_{i}^{e} \beta_{i}^{e} b_{i+1}^{e} b_{i+2}^{e} \ldots b_{H}^{e}$ \\
$B_{i, 4}^{e}$ & $a_{i}^{e} \ldots a_{H}^{e} \cdot \bar{b}_{1}^{e} \ldots \bar{b}_{i-1}^{e} \beta_{i}^{e} \bar{b}_{i}^{e} b_{i+1}^{e} b_{i+2}^{e} \ldots b_{H}^{e}$ \\
after $B_{H, 4}^{e}$ & $\bullet \bar{b}_{1}^{e} \ldots \bar{b}_{H}^{e}$ \\
\hline
\end{tabular}

(d) In each block, pages associated with $e_{1}$ are requested first, then pages associated with $e_{2}$ are requested and so on up to $e_{m}$.

(e) For each block $B$ and each edge $e$, all requests on $a^{e}$-pages and $\bar{b}^{e}$-pages in $B$ precede all requests on $\bar{a}^{e}$-pages and $b^{e}$-pages in $B$.

(f) Let $e=\{u, v\}$ be an edge and $p$ an $\alpha^{e}$-page or $\beta^{e}$-page. Let $B$ be the first block and $\bar{B}$ the last block where $p$ is requested. Then $B$ and $\bar{B}$ are either both in the $u$-phase or both in the $v$-phase. Furthermore, no other page of size three associated with $e$ is requested in $B, \bar{B}$, or any block between them.

Lemma 3.3. The instance from Reduction 2.1 satisfies Properties 3.2.

Proof. All properties (a), (b), (c), (d), (f) follow directly from Reduction 2.1 and the subsequent observations. To prove (e), recall that the pages associated with an edge $e$ requested in a particular block always follow the ordering (1). We need to verify that when the page $a_{i}^{e}$ is requested, no page $\bar{a}_{j}^{e}$ for $j \leq i$ is requested and that when the page $\bar{b}_{i}^{e}$ is requested, no $\bar{a}^{e}$-page and no page $b_{j}^{e}$ for $j \leq i$ is requested. This can be seen easily when we explicitly write down the request sequences for each kind of block, see Table 2.

For the following claims, let $\mathcal{T}_{G}$ be an instance fulfilling Properties 3.2. We fix a service of $\mathcal{T}_{G}$ with the total savings of at least $(d-1) m H$.

Let $\mathcal{B}$ be the set of all blocks and $\overline{\mathcal{B}}$ the set of all blocks except for the initial and final one. For a block $B$, we denote the block immediately following it by $B^{\prime}$.

We define two useful values characterizing the service for the block $B: \delta_{B}=$ $m H-s_{B}$ (the number of free slots for edge-pages at the start of the service of the block) and $\gamma_{B}^{e}=\left|s_{B^{\prime}}^{e}-s_{B}^{e}\right|$ (the change of the number of slots occupied by pages associated with $e$ after requests from this block are served).

The first easy lemma says that only a small number of blocks can start with some free slots in the cache.

Lemma 3.4. When summing over all blocks except for the initial one

$$
\sum_{B \in \mathcal{B} \backslash\{I\}} \delta_{B} \leq n .
$$


Proof. Using the property (b) and $s_{I}=0$, the savings on edge-pages are

$$
\sum_{B \in \mathcal{B} \backslash\{I\}} s_{B}=(d-1) m H-\sum_{B \in \mathcal{B} \backslash\{I\}} \delta_{B} .
$$

The total savings are assumed to be at least $(d-1) m H$. Due to the property (a), the savings on vertex-pages are at most $n$. Claim of the lemma follows.

The second lemma states that the number of slots occupied by pages associated with a given edge does not change much during the whole service.

Lemma 3.5. For each edge $e \in E$,

$$
\sum_{B \in \overline{\mathcal{B}}} \gamma_{B}^{e} \leq 6 n
$$

Proof. Let us use the notation $S_{\bar{B}}^{\leq k}=S_{B}^{e_{1}} \cup \cdots \cup S_{B}^{e_{k}}$ and $s_{\bar{B}}^{\leq k}=\left|S_{\bar{B}}^{\leq k}\right|$. First, we shall prove for each $k \leq m$

$$
\sum_{B \in \overline{\mathcal{B}}}\left|s_{\bar{B}^{\prime}}^{\leq k}-s_{\bar{B}}^{\leq k}\right| \leq 3 n
$$

Let $\mathcal{P}$ denote the set of all blocks $B$ from $\overline{\mathcal{B}}$ satisfying $s_{\bar{B}^{\prime}}^{\leq k}-s_{\bar{B}}^{\leq k} \geq 0$ and let $\mathcal{N}$ denote the set of all the remaining blocks from $\overline{\mathcal{B}}$.

As a consequence of the property (c), we get $s_{I^{\prime}}^{\leq k} \in\left[k H-\delta_{I^{\prime}}, k H\right]$ and $s_{F}^{\leq k} \in\left[k H-\delta_{F}, k H\right]$. So we obtain the inequality

$$
s_{\bar{F}}^{\leq k}-s_{I^{\prime}}^{\leq k} \geq-\delta_{F} .
$$

We claim $s_{\bar{B}^{\prime}}^{\leq k}-s_{\bar{B}}^{\leq k} \leq \delta_{B}$ for each $B \in \overline{\mathcal{B}}$. We assume for a contradiction $s_{B^{\prime}}^{\leq k}-s_{B}^{\leq k}>\delta_{B}$ for some block $B$. We use the property (d). Then after processing the edge $e_{k}$ in $B$, the number of edge-pages in the cache is $\left(s_{B}-s_{B}^{\leq k}\right)+s_{B^{\prime}}^{\leq k}>s_{B}+$ $\delta_{B}=m H$. But more than $m H$ edge-pages in the cache means a contradiction.

The summation over all blocks from $\mathcal{P}$ gives us the first bound

$$
\sum_{B \in \mathcal{P}}\left(s_{B^{\prime}}^{\leq k}-s_{B}^{\leq k}\right) \leq \sum_{B \in \mathcal{P}} \delta_{B} \leq n .
$$

Using the fact $\mathcal{P} \dot{\cup} \mathcal{N}=\overline{\mathcal{B}}$ and (3), we have

$$
\sum_{B \in \mathcal{P}}\left(s_{B^{\prime}}^{\leq k}-s_{B}^{\leq k}\right)+\sum_{B \in \mathcal{N}}\left(s_{B^{\prime}}^{\leq k}-s_{B}^{\leq k}\right)=s \frac{\leq k}{F}-s_{I^{\prime}}^{\leq k} \geq-\delta_{F} ;
$$

together with (4), we obtain the second bound

$$
-\sum_{B \in \mathcal{N}}\left(s_{B^{\prime}}^{\leq k}-s_{B}^{\leq k}\right) \leq \sum_{B \in \mathcal{P}}\left(s_{B^{\prime}}^{\leq k}-s_{B}^{\leq k}\right)+\delta_{F} \leq 2 n .
$$


Combining the bounds (4) and (5), we prove (2)

$$
\sum_{B \in \overline{\mathcal{B}}}\left|s_{B^{\prime}}^{\leq k}-s_{\bar{B}}^{\leq k}\right|=\sum_{B \in \mathcal{P}}\left(s_{B^{\prime}}^{\leq k}-s_{\bar{B}}^{\leq k}\right)-\sum_{B \in \mathcal{N}}\left(s_{B^{\prime}}^{\leq k}-s_{B}^{\leq k}\right) \leq n+2 n=3 n .
$$

For the edge $e_{1}$, the claim of this lemma is weaker than (2) because $\gamma_{B}^{e_{1}}=$ $\left|s_{B^{\prime}}^{e_{1}}-s_{B}^{e_{1}}\right|$. Proving our lemma for $e_{k}$ when $k>1$ is just a matter of using (2) for $k-1$ and $k$ together with the formula $|x-y| \leq|x|+|y|$ :

$$
\sum_{B \in \overline{\mathcal{B}}} \gamma_{B}^{e_{k}} \leq \sum_{B \in \overline{\mathcal{B}}}\left|s_{B^{\prime}}^{\leq k}-s_{\bar{B}}^{\leq k}\right|+\sum_{B \in \overline{\mathcal{B}}}\left|s_{\bar{B}^{\prime}}^{\leq k-1}-s_{B}^{\leq k-1}\right| \leq 3 n+3 n=6 n
$$

For the rest of the proof, we set $H=6 m n+3 n+1$. This enables us to show that the fixed service must cache some of the pages of size three.

Lemma 3.6. For each edge $e \in E$, there is a block $B$ such that some $\alpha^{e}$-page or $\beta^{e}$-page is in $S_{B}$ and $\delta_{B}=0$.

Proof. Fix an edge $e=e_{k}$. For each block $B$, we define

$$
\varepsilon_{B}=\text { number of } \alpha^{e} \text {-pages and } \beta^{e} \text {-pages in } S_{B} \text {. }
$$

Observe that due to the property (f), $\varepsilon_{B}$ is always one or zero. We use a potential function

$$
\Phi_{B}=\text { number of } a^{e} \text {-pages and } \bar{b}^{e} \text {-pages in } S_{B} \text {. }
$$

Because there are only $\bar{a}$-pages in the initial block and only $\bar{b}$-pages in the final block (property (c)), we know

$$
\Phi_{I^{\prime}}=0 \quad \text { and } \quad \Phi_{F} \geq H-\delta_{F} .
$$

Now we bound the increase of the potential function as

$$
\Phi_{B^{\prime}}-\Phi_{B} \leq \delta_{B}+\sum_{\ell=1}^{k-1} \gamma_{B}^{e_{\ell}}+\varepsilon_{B}
$$

To justify this bound, we fix a block $B$ and look at the cache state after requests on edges $e_{1}, \ldots, e_{k-1}$ are processed. How many free slots there can be in the cache? There are initial $\delta_{B}$ free slots in the beginning of the block $B$, and the number of free slots can be further increased when the number of pages in the cache associated with $e_{1}, \ldots, e_{k-1}$ decreases. This increase can be naturally bounded by $\sum_{\ell=1}^{k-1} \gamma_{B}^{e_{\ell}}$. Therefore, the number of free slots in the cache is at most $\delta_{B}+\sum_{\ell=1}^{k-1} \gamma_{B}^{e_{\ell}}$.

Because of the property (e), the number of cached $a^{e}$-pages and $\bar{b}^{e}$-pages can only increase by using the free cache space or caching new pages instead of $\alpha^{e}$-pages and $\beta^{e}$-pages. We already bounded the number of free slots and $\varepsilon_{B}$ is 
a natural bound for the increase gained on $\alpha^{e}$-pages and $\beta^{e}$-pages. Thus, the bound (7) is correct.

Summing (7) over all $B \in \overline{\mathcal{B}}$, we have

$$
\Phi_{F}-\Phi_{I^{\prime}}=\sum_{B \in \overline{\mathcal{B}}}\left(\Phi_{B^{\prime}}-\Phi_{B}\right) \leq \sum_{B \in \overline{\mathcal{B}}}\left(\delta_{B}+\sum_{\ell=1}^{k-1} \gamma_{B}^{e_{\ell}}+\varepsilon_{B}\right)
$$

which we combine with (6) into

$$
H-\delta_{F} \leq \sum_{B \in \overline{\mathcal{B}}}\left(\delta_{B}+\sum_{\ell=1}^{k-1} \gamma_{B}^{e_{\ell}}+\varepsilon_{B}\right)
$$

and use Lemmata 3.4 and 3.5 to bound $\sum \varepsilon_{B}$ as

$$
\begin{aligned}
\sum_{B \in \overline{\mathcal{B}}} \varepsilon_{B} & \geq H-\delta_{F}-\sum_{B \in \overline{\mathcal{B}}}\left(\delta_{B}+\sum_{\ell=1}^{k-1} \gamma_{B}^{e_{\ell}}\right) \\
& \geq H-n-n-(k-1) 6 n \\
& \geq H-6 m n-2 n=n+1 .
\end{aligned}
$$

As there is at most one page of size three requested in each block (property (f)), the inequality $\sum \varepsilon_{B} \geq n+1$ implies that there are at least $n+1$ blocks where an $\alpha^{e}$-page or a $\beta^{e}$-page is cached. At most $n$ blocks have $\delta_{B}$ non-zero (Lemma 3.4); we are done.

We are ready to complete the proof of the harder direction.

Lemma 3.7. Suppose that there exists a service of $\mathcal{T}_{G}$ with the total savings of at least $(d-1) m H+K$. Then the graph $G$ has an independent set $W$ of cardinality $K$.

Proof. Let $W$ be a set of $K$ vertices such that the corresponding page $p_{v}$ is cached between its two requests. (There are at least $K$ of them because the maximal savings on edge-pages are $(d-1) m H$.)

Consider an arbitrary edge $e=\{u, v\}$. Due to Lemma 3.6, there exists a block $B$ such that $\delta_{B}=0$ and some $\alpha^{e}$-page or $\beta^{e}$-page is cached in the beginning of the block. This block $B$ is either in the $u$-phase or in the $v$-phase, because of the statement of the property (f). This means that at least one of the two pages $p_{u}$ and $p_{v}$ is not cached between its two requests, because the cache is full. As a consequence, the set $W$ is indeed independent.

The value of $H$ was set to $6 m n+3 n+1$, therefore Reduction 2.1 is indeed polynomial. Lemmata 3.1, 3.3 and 3.7 together imply that there is an independent set of cardinality $K$ in $G$ if and only if there is a service of the instance $\mathcal{I}_{G}$ with the total savings of at least $(d-1) m H+K$. We showed that the problem 3CACHING(FAULT, OPTIONAL) is indeed strongly NP-hard. 


\section{Bit Model}

In this section, we show how to modify the proof for the fault model from the previous sections so that it works as a proof for the bit model as well.

Reduction 4.1. Let $G$ be a graph and $\mathcal{I}_{G}$ the corresponding instance of the problem 3CACHING(FAUlt, OPTIONAL) from Reduction 2.1. Then the modified

instance $\widetilde{\mathcal{I}}_{G}$ is an instance of 3 CACHING(BIT, OPTIONAL) with the same cache size and the same set of pages with the same sizes.

The structure of phases and requests on vertex-pages is also preserved. The blocks from $\mathcal{I}_{G}$ are also used, but between each pair of consecutive blocks there are five new blocks inserted. Let $B$ and $B^{\prime}$ be two consecutive blocks. Between $B$ and $B^{\prime}$ we insert five new blocks $B_{(1)}, \ldots, B_{(5)}$ with the following requests

$-B_{(1)}$ : do not request anything;

$-B_{(2)}$ : request all pages of size two that are requested both in $B$ and $B^{\prime}$;

$-B_{(3)}$ : request a page (there is either one or none) of size three that is requested both in $B$ and $B^{\prime}$;

$-B_{(4)}$ : request all pages of size two that are requested both in $B$ and $B^{\prime}$;

$-B_{(5)}$ : do not request anything.

See Fig. 5 for an example. In each new block, the order of chosen requests is the same as in $B$ (which is the same as in $B^{\prime}$, as both follow the same ordering of edges (1)). The new instance has the total of $\tilde{d}=d+5(d-1)=6 d-5$ blocks. This time we prove that the maximal total savings are $(\tilde{d}-1) m H+K$ where $K$ is the cardinality of the maximum independent set in $G$.

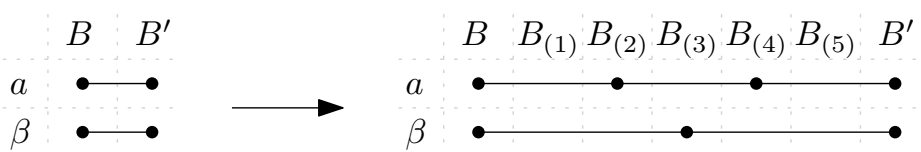

Fig. 5. The modification of the instance for a page $a$ of size two and a page $\beta$ of size three

Lemma 4.2. Suppose that the graph $G$ has an independent set $W$ of cardinality $K$. Then there exists a service of the modified instance $\widetilde{\mathcal{I}}_{G}$ with the total savings of at least $(\tilde{d}-1) m H+K$.

Proof. We consider the service of the original instance $\mathcal{I}_{G}$ described in the proof of Lemma 3.1 and modify it so it becomes a service of the modified instance.

In the new service, vertex-pages are served the same way as in the original service. The savings on vertex-pages are thus again $K$.

For each pair of consecutive blocks $B$ and $B^{\prime}$, each page kept in the cache between $B$ and $B^{\prime}$ in the original service is kept in the cache in the new service for the whole time between $B$ and $B^{\prime}$ (it spans over seven blocks now). For a page of size two, savings of two are incurred three times. For a page of size three, savings of three are incurred twice. On each page in the new service we save six instead of one. Therefore, the total savings on edge-pages are $6(d-1) m H=(\tilde{d}-1) m H$.

The total savings are $(\tilde{d}-1) m H+K$. 
Lemma 4.3. Suppose that there exists a service of the modified instance $\widetilde{\mathcal{I}}_{G}$ with the total savings of at least $(\tilde{d}-1) m H+K$. Then the graph $G$ has an independent set $W$ of cardinality $K$.

Proof. This lemma is the same as Lemma 3.7. We just need to verify that the modified instance fulfills Properties 3.2.

To prove that the property (b) is preserved, we observe that each two consecutive requests on a page of size two are separated by exactly one block where the page is not requested. Consequently, when there are savings of two on a request on the page of size two, we assign savings of one to the block where the savings were incurred and savings of one to the previous block. Similarly, each pair of consecutive requests on a page of size three is separated by exactly two blocks where the page is not requested. When there are savings of three on a request on the page of size three, we assign savings of one to the block where the savings were incurred and savings of one to each of the two previous blocks. As a consequence, the total savings gained on edge-pages may indeed be computed as $\sum s_{B}$.

The property (a) is preserved because the requests on vertex-pages are the same in both instances. The property (c) is preserved because the initial and final blocks are the same in both instances.

Each sequence of requests in a block of the modified instance $\widetilde{\mathcal{I}}_{G}$ is either the same or a subsequence of the sequence in a block in the original instance. Therefore, the properties (d), (e) and (f) are preserved as well.

Lemmata 4.2 and 4.3 imply that we have a valid polynomial-time reduction and so the problem 3CACHING(BIT,OPTIONAL) is strongly NP-hard.

\section{Forced Policy}

Theorem 5.1. Both the problem 3CACHING(FAULT,FORCED) and the problem 3CACHING(BIT,FORCED) are strongly NP-hard.

Proof. For both the fault model and the bit model, we show a polynomialtime reduction from caching with optional policy to the corresponding variant of caching with the forced policy. Let us have an instance of caching with the optional policy with the cache size $C$ and the request sequence $\rho=r_{1} \ldots r_{n}$; let $M$ be the maximal size of a page in $\rho$ (in our previous reductions, $M=3$ ).

We create an instance of caching with the forced policy. The cache size is $C^{\prime}=C+M$. The request sequence is $\rho^{\prime}=r_{1} q_{1} r_{2} q_{2} \ldots r_{n} q_{n}$ where $q_{1}, \ldots, q_{n}$ are requests to $n$ different pages that do not appear in $\rho$ and have size $M$. The costs of the new pages are one in the fault model and $M$ in the bit model.

We claim that there is a service of the optional instance with savings $S$ if and only if there is a service of the forced instance with savings $S$.

$\Rightarrow$ We serve the requests on original pages the same way as in the optional instance. The cache is larger by $M$ which is the size of the largest page. Thus, pages that were not loaded into the cache because of the optional policy fit in 
there; we can load them and immediately evict them. New pages fit into the cache as well and we also load them and immediately evict them. This way we have the same savings as in the optional instance.

$\Leftarrow$ We construct a service for the optional instance: For each $i$, when serving $r_{i}$ we consider the evictions done when serving $r_{i}$ and $q_{i}$ of the forced instance. If a page requested before $r_{i}$ is evicted, we evict it as well. If a page requested by $r_{i}$ is evicted, we do not cache it at all. Because the page requested by $q_{i}$ has size $M$, the original pages occupy at most $C$ slots in the cache when $q_{i}$ is served. This way we obtain a service of the optional instance with the same savings.

Using the strong NP-hardness of the problems 3CACHING(FAULT, OPTIONAL) and 3CACHING(BIT, OPTIONAL) proven in Sections 2 and 3 and the observation that the reduction preserves the maximal size of a page, we obtain the strong NP-hardness of 3CACHING(FAULT, FORCED) and 3CACHING(BIT, FORCED).

\section{References}

1. Adamaszek, A., Czumaj, A., Englert, M., Räcke, H.: An $O(\log k)$-competitive algorithm for generalized caching. In: Proceedings of the 23rd Annual ACM-SIAM Symposium on Discrete Algorithms (SODA). pp. 1681-1689. SIAM (2012), http: //dl.acm.org/citation. cfm?id=2095116. 2095249

2. Albers, S., Arora, S., Khanna, S.: Page replacement for general caching problems. In: Proceedings of the 10th Annual ACM-SIAM Symposium on Discrete Algorithms. pp. 31-40 (1999), http://dl .acm.org/citation. cfm?id=314500.314528

3. Bansal, N., Friggstad, Z., Khandekar, R., Salavatipour, M.R.: A logarithmic approximation for unsplittable flow on line graphs. ACM Transactions on Algorithms 10(1), 1 (2014), http://doi.acm.org/10.1145/2532645, a preliminary version appeared at SODA 2009.

4. Bar-Noy, A., Bar-Yehuda, R., Freund, A., Naor, J., Schieber, B.: A unified approach to approximating resource allocation and scheduling. Journal of the ACM 48(5), 1069-1090 (2001), http://doi.acm.org/10.1145/502102.502107, a preliminary version appeared at STOC 2000.

5. Belady, L.A.: A study of replacement algorithms for a virtual-storage computer. IBM Systems Journal 5(2), 78-101 (Jun 1966), http://dx.doi.org/10.1147/sj . 52.0078

6. Borodin, A., El-Yaniv, R.: Online computation and competitive analysis. Cambridge University Press (1998)

7. Chrobak, M., Karloff, H.J., Payne, T.H., Vishwanathan, S.: New results on server problems. SIAM Journal on Discrete Mathematics 4(2), 172-181 (1991), http: //dx.doi.org/10.1137/0404017, a preliminary version appeared at SODA 1990.

8. Chrobak, M., Woeginger, G.J., Makino, K., Xu, H.: Caching is hard - even in the fault model. Algorithmica 63(4), 781-794 (2012), http://dx.doi.org/10.1007/ s00453-011-9502-9, a preliminary version appeared at ESA 2010.

9. Irani, S.: Page replacement with multi-size pages and applications to web caching. Algorithmica 33(3), 384-409 (2002), http://dx.doi.org/10.1007/ s00453-001-0125-4, a preliminary version appeared at STOC 1997.

10. Young, N.E.: On-line file caching. Algorithmica 33(3), 371-383 (2002), http://dx . doi.org/10.1007/s00453-001-0124-5, a preliminary version appeared at SODA 1998. 


\section{A The Simple Proof}

In this appendix, we present a simple variant of the proof for the almost-fault model with two distinct costs. This completes the sketch of the proof presented at the end of Section 2. We present it with a complete description of the simplified reduction, so that it can be read independently of the rest of the paper. This appendix can therefore serve as a short proof of the hardness of general caching.

Theorem A.1. General caching is strongly NP-hard, even in the case when page sizes are limited to $\{1,2,3\}$ and there are only two distinct fault costs.

We prove the theorem for the optional policy. It is easy to obtain the theorem also for the forced policy the same way as in the proof of Theorem 5.1.

\section{The Reduction}

The reduction described here will be equivalent to Reduction 2.1 with $H=1$ and the fault cost of each vertex-page set to $1 /(n+1)$.

Suppose we have a graph $G=(V, E)$ with $n$ nodes and $m$ edges. We construct an instance of general caching whose optimal solution encodes a maximum independent set in $G$. Fix an arbitrary numbering of edges $e_{1}, \ldots, e_{m}$.

The cache size is $C=2 m+1$. For each vertex $v$, we have a vertex-page $p_{v}$ with size one and cost $1 /(n+1)$. For each edge $e$, we have six associated edgepages $a^{e}, \bar{a}^{e}, \alpha^{e}, b^{e}, \bar{b}^{e}, \beta^{e}$; all have cost one, pages $\alpha^{e}, \beta^{e}$ have size three and the remaining pages have size two.

The request sequence is organized in phases and blocks. There is one phase for each vertex. In each phase, there are two adjacent blocks associated with every edge $e$ incident with $v$; the incident edges are processed in an arbitrary order. In addition, there is one initial block $I$ before all phases and one final block $F$ after all phases. Altogether, there are $d=4 m+2$ blocks. There are four blocks associated with each edge $e$; denote them $B_{1}^{e}, B_{2}^{e}, B_{3}^{e}, B_{4}^{e}$, in the order as they appear in the request sequence.

For each $v \in V$, the associated page $p_{v}$ is requested exactly twice, right before the beginning of the $v$-phase and right after the end of the $v$-phase; these requests do not belong to any phase. An example of the structure of phases and blocks is given in Fig. 6.

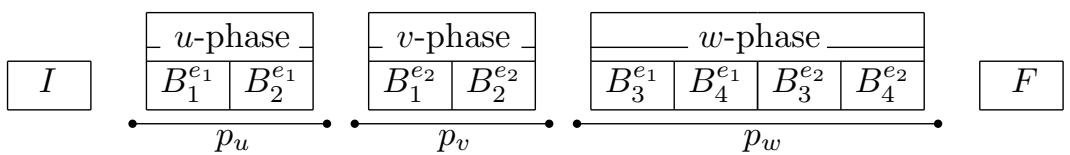

Fig. 6. An example of phases, blocks and requests on vertex-pages for a graph with three vertices $u, v, w$ and two edges $e_{1}=\{u, w\}, e_{2}=\{v, w\}$ when $H=2$

Even though each block is associated with some fixed edge, it contains one or more requests to the associated pages for every edge $e$. In each block, we process the edges $e_{1}, \ldots, e_{m}$ in this order. For each edge $e$, we make one or more requests to the associated pages as follows. If the current block is: 
- before $B_{1}^{e}$ : request $\bar{a}^{e}$;

- $B_{1}^{e}$ : request $\bar{a}^{e}, \alpha^{e}$, and $b^{e}$;

- $B_{2}^{e}$ : request $\alpha^{e}, a^{e}$, and $b^{e}$;

- after $B_{2}^{e}$ and before $B_{3}^{e}$ : request $a^{e}$ and $b^{e}$;

- $B_{3}^{e}$ : request $a^{e}, b^{e}$, and $\beta^{e}$;

- $B_{4}^{e}$ : request $a^{e}, \beta^{e}$, and $\bar{b}^{e}$;

- after $B_{4}^{e}$ : request $\bar{b}^{e}$.

Fig. 7 shows an example of the requests on edge-pages associated with one particular edge.

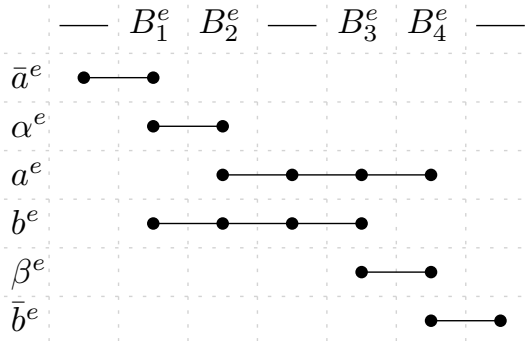

Fig. 7. Requests on all pages associated with the edge $e$. Each column represents some block(s). The four labelled columns represent the blocks in the heading, the first column represents every block before $B_{1}^{e}$, the middle column represents every block between $B_{3}^{e}$ and $B_{4}^{e}$, and the last column represents every block after $B_{4}^{e}$. The requests in one column are ordered from top to bottom.

\section{Proof of Correctness}

Instead of minimizing the service cost, we maximize the savings compared to the service which does not use the cache at all. This is clearly equivalent when considering the decision version of the problem.

Without loss of generality, we assume that any page is brought into the cache only immediately before a request to that page and removed from the cache only immediately after a request to that page; furthermore, at the beginning and at the end the cache is empty. I.e., a page may be in the cache only between two consecutive requests to this page, and either it is in the cache for the whole interval or not at all.

Each page of size three is requested only twice in two consecutive blocks, and these blocks are distinct for all pages of size three. Thus, a service of edge-pages is valid if and only if at each time, at most $m$ edge-pages are in the cache. It is thus convenient to think of the cache as of $m$ slots for edge-pages.

Each vertex-page is requested twice. Thus, the savings on the $n$ vertex-pages are at most $n /(n+1)<1$. Since all edge-pages have cost one, the optimal service must serve them optimally. Furthermore, a vertex-page can be cached if and only if during the phase it never happens that at the same time all slots for edge-pages are full and a page of size three is cached. 
Let $S_{B}$ denote the set of all edge-pages cached at the beginning of the block $B$ and let $s_{B}=\left|S_{B}\right|$. Now observe that each edge-page is requested only in a contiguous segment of blocks, once in each block. It follows that the total savings on edge-pages are equal to $\sum_{B} s_{B}$ where the sum is over all blocks. In particular, the maximal possible savings on the edge-pages are $(d-1) m$, using the fact that $S_{I}$ is empty.

We prove that there is a service with the total savings of at least $(d-1) m+$ $K /(n+1)$ if and only if there is an independent set of size $K$ in $G$. First the easy direction.

Lemma A.2. Suppose that $G$ has an independent set $W$ of size $K$. Then there exists a service with the total savings of $(d-1) m+K /(n+1)$.

Proof. For any $e$, denote $e=u v$ so that $u$ precedes $v$ in the ordering of phases. If $u \in W$, we keep $\bar{a}^{e}, b^{e}, \bar{b}^{e}$ and $\beta^{e}$ in the cache from the first to the last request on each page, and we do not cache $a^{e}$ and $\alpha^{e}$ at any time. Otherwise we cache $\bar{b}^{e}, a^{e}, \bar{a}^{e}$ and $\alpha^{e}$, and do not cache $b^{e}$ and $\beta^{e}$ at any time. In both cases, at each time at most one page associated with $e$ is in the cache and the savings on those pages is $(d-1) m$. See Fig. 8 for an illustration.

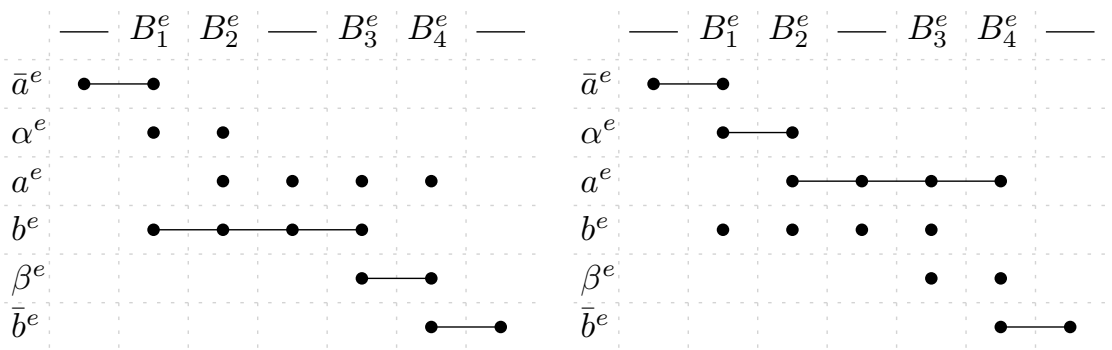

Fig. 8. The two ways of caching in Lemma A.2

For any $v \in W$, we cache $p_{v}$ between its two requests. To check that this is a valid service, observe that if $v \in W$, then during the corresponding phase no page of size three is cached. Thus the page $p_{v}$ always fits in the cache together with at most $m$ pages of size two.

Now we prove the converse in a sequence of claims. Fix a valid service with savings at least $(d-1) \mathrm{m}$. For a block $B$, let $B^{\prime}$ denote the following block.

Claim A.3. For any block $B$, with the exception of $B=I$, we have $s_{B}=m$.

Proof. For each $B \neq I$ we have $s_{B} \leq m$. Because $s_{I}=0$, the total savings on edge-pages are $\sum_{B} s_{B} \leq(d-1) m$. We need an equality.

We now prove that each edge occupies exactly one slot during the service.

Claim A.4. For any block $B \neq I$ and for any $e, S_{B}$ contains exactly one page associated with $e$. 
Proof. Let us use the notation $S_{\bar{B}}^{\leq k}=S_{B}^{e_{1}} \cup \cdots \cup S_{B}^{e_{k}}$ and $s_{B}^{\leq k}=\left|S_{\bar{B}}^{\leq k}\right|$. First, we shall prove for each $k \leq m$

$$
s_{B}^{\leq k}=k .
$$

This is true for $B=F$, as only the $m$ edge-pages $\bar{b}^{e}$ can be cached there, and by the previous claim all of them are indeed cached. Similarly for $B=I^{\prime}$ (i.e., immediately following the initial block).

If (8) is not true, then for some $k$ and $B \notin\{I, F\}$ we have $s \frac{<k}{B}<s \frac{\leq k}{B^{\prime}}$. Then after processing the edge $e_{k}$ in the block $B$ we have in the cache all the pages in $\left(S_{B} \backslash S_{\bar{B}}^{\leq k}\right) \cup S_{\bar{B}^{\prime}}^{\leq k}$. Their number is $\left(m-s_{B}^{\leq k}\right)+s_{B^{\prime}}^{\leq k}>m$, a contradiction.

The statement of the claim is an immediate consequence of (8).

Claim A.5. For any edge $e$, at least one of the pages $\alpha^{e}$ and $\beta^{e}$ is cached between its two requests.

Proof. Assume that none of the two pages is cached. It follows from the previous claim that $b^{e} \in S_{B_{2}^{e}}$, as at this point $\alpha^{e}$ and $b^{e}$ are the only pages associated with $e$ that can be cached. Similarly, $a^{e} \in S_{B_{4}^{e}}$.

It follows that there exists a block $B$ between $B_{1}^{e}$ and $B_{4}^{e}$ such that $S_{B}$ contains the page $b^{e}$ and $S_{B^{\prime}}$ contains the page $a^{e}$. However, in $B$, the page $a^{e}$ is requested before the page $b^{e}$. Thus at the point between the two requests, the cache contains two pages associated with $e$, plus one page associated with every other edge, the total of $m+1$ pages, a contradiction.

Now we are ready to complete this direction.

Lemma A.6. Suppose that there exists a valid service with the total savings of $(d-1) m+K /(n+1)$. Then $G$ has an independent set $W$ of size $K$.

Proof. Let $W$ be the set of all $v$ such that $p_{v}$ is cached between its two requests. The total savings imply that $|W|=K$.

Now we claim that $W$ is independent. Suppose not, let $e=u v$ be an edge with $u, v \in W$. Then $p_{u}$ and $p_{v}$ are cached in the corresponding phases. Thus neither $\alpha^{e}$ nor $\beta^{e}$ can be cached, since together with other $m-1$ requests of size 2 associated with the remaining edges, the cache size needed would be $2 m+2$. However, this contradicts the last claim.

Lemmata A.2 and A.6 together show that we constructed a valid polynomialtime reduction from the problem of independent set to general caching. Therefore, Theorem A.1 is proven. 